\title{
Cataract Surgical Planning Using Online Software vs Traditional Methods: A Time/Motion and Quality of Care Study
}

\author{
Tarika Gujral ${ }^{1}$ \\ John Hovanesian (iD) ${ }^{2}$ \\ 'College of Letters and Science, \\ University of California, Los Angeles, CA, \\ USA; ${ }^{2}$ Cornea/Cataract, Harvard Eye \\ Associates, Laguna Hills, CA, USA
}

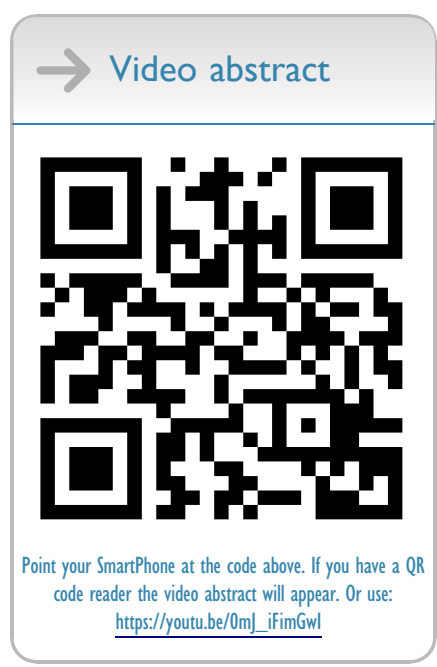

Correspondence: John Hovanesian Harvard Eye Associates, 2396 I Calle Magdalena, Suite 300, Laguna Hills, CA, 92653, USA

Tel + I 9499512020

Email jhovanesian@harvardeye.com
Purpose: The objective of this study is to compare two methods of preoperative planning for placement of intraocular implants: traditional paper documentation/electronic scans versus a novel online planning software. The aim was to compare the time required and number of data points manually transcribed with each method and to explore whether differences in surgical accuracy could be identified between the two methods in a small sample of 40 patients.

Methods: In this study, preoperative planning was performed twice for all enrolled patients: once through the traditional method and once using an online planning software system. The total time spent and number of data points manually transferred were recorded for each method.

Results: Of the 40 patients enrolled, the mean total surgical planning time was $239 \pm 190$ seconds for the traditional method vs $63 \pm 31$ seconds with the online planning software $(\mathrm{P}<0.00001)$. The mean number of data points transcribed was $7.2 \pm 7.2$ for the traditional method vs $0.9 \pm 1.7$ with the online planning software $(\mathrm{P}<0.0000001)$. No statistically significant differences were noted in terms of accuracy of prediction of the spherical equivalent surgical outcome.

Conclusion: In comparison to traditional methods, a significant reduction in time required for surgical planning and the manual transcription of data points was noted when a comprehensive online surgical planning software was used. This has important implications for the efficiency and likely the safety of surgical planning.

Keywords: biometry, accuracy, intraocular lens; errors

\section{Introduction}

Cataract is one of the leading correctable causes of blindness, affecting about 24.4 million Americans over forty. ${ }^{1}$ Cataracts develop when proteins accumulate in the lens, eventually resulting in cloudiness and decreased vision. This occurs naturally as one ages, and may be exacerbated through trauma, radiation, medications, and systemic diseases. Symptoms experienced are fading of colors, glare, diplopia, sensitivity to bright light, and impaired night vision. Without treatment, cataracts continue to progress, eventually impacting quality of life. ${ }^{2}$ Fortunately, vision loss due to cataracts can be restored via corrective surgery.

Cataract surgery requires extensive preoperative planning to select an intraocular lens (IOL) of proper power for the eye undergoing surgery. To perform this calculation, a detailed ocular exam is required, and ocular biometry, including axial 
length, anterior chamber depth, lens thickness, corneal white-to-white measurements, and keratometry must be performed. The required formulas for performing IOL calculations are often integrated into the same diagnostic instruments that perform measurements, which create a report, allowing surgeons to choose a lens power for each IOL model being considered to achieve the desired spherical equivalent refraction. ${ }^{3}$ However, patients who have undergone prior refractive surgery and those who require toric IOL correction or incisional keratotomy may require additional calculations performed with special formulas, such as the Barrett True K or the Barrett Toric Formula, and these formulas do not uniformly reside on biometry machines, necessitating transfer of the data to a separate system for surgical planning. ${ }^{4}$ This transfer of data introduces the possibility of transcription errors and consumes significant time. Considering that errors in transcribing numbers from one platform to another are possible, manual transcription of information critical to the refractive outcome is certainly not ideal.

A thorough preoperative workup also requires evaluation of tear film and ocular surface, endothelial cell count, optical coherence tomography (OCT) of the macula, and other diagnostic tests. To review these documents during surgical planning generally requires accessing the diagnostic images in a paper chart or through a digital image management system that again is a separate system from that used for lens power calculation or toric calculation.

Therefore, for calculations for a single eye, the surgeon might need to access the report from the optical biometer, enter information into online calculators (one for toric and one or more for spherical equivalent IOL power), view the images from corneal topography and OCT scan, and view diagnosis information from the medical record - six or more sources - for a single surgical plan.

As a result of the complex and error-prone nature of this process, medical facilities are beginning to implement single, all-encompassing online software to mitigate time and error associated with manual transfer of data points. In this study, we evaluated an online surgical planning software in which data and formulas required for cataract preoperative planning are stored on a single cloud-based platform. This software interfaces with both electronic medical record (EMR) systems and diagnostic devices, synthesizing the data obtained to present a single, simple user interface to a surgeon in order to enhance efficiency of preoperative planning. The objective of this study was to evaluate the time required to plan surgery by each method and to compare the number of data points manually transcribed with online planning software and compare it with traditional preoperative planning methods. As an exploratory endpoint, surgical accuracy was also analyzed by evaluating which planning method resulted in a more accurate surgical outcome.

\section{Materials and Methods}

This single-center, prospective, open-label, controlled study was conducted with patients of a single surgeon (JAH). An informed consent and HIPAA waiver were obtained from Aspire IRB (Santee, CA) since the study involved no intervention, questions, or measurements other than those routinely performed during cataract surgery. This study was conducted under the protocols of the Declaration of Helsinki and was registered at clinicaltrials. gov (NCT04337892). All appropriate measures were taken to ensure anonymity of patient and investigator's personal data, which was recorded in compliance with all applicable laws and regulations.

The inclusion criteria for this study allowed patients over the age of eighteen with upcoming cataract surgery. Forty consecutive patients were enrolled with an aim to achieve $25 \%$ of enrolled eyes with prior keratorefractive surgery and $25 \%$ who required astigmatic planning (arcuate incision or toric IOL).

Patients with visually significant comorbidities (corneal, retina, optic nerve disease) that could significantly affect the ability to have accurate preoperative biometry or postoperative refraction were excluded. Patients with surgical complications, either during or after surgery, such as capsular tears, iris trauma, decentered IOLs, cystoid macular edema, etc. that would interfere with postoperative refraction were also excluded. Additionally, the study excluded patients with grade 2 or greater posterior capsule opacification at the time of postoperative refraction at the final visit.

If both eyes were to undergo surgery, the eye scheduled first was selected for data collection. All study eyes underwent preoperative planning twice: once with the surgeon using traditional EHR and scanned paper methods and a second time using an online planning software (Veracity Surgical, Zeiss, Oberkochen, Germany). The latter system connected digitally to diagnostic instruments in the clinic, including an optical biometer (IOLmaster, Zeiss), corneal topographer (Atlas, Zeiss), and OCT (Stratus, Zeiss). The software automatically imported biometry information as structured data. The surgeon used the 
graphical user interface to select an IOL calculation formula and lens power.

To evaluate the primary outcome measure, time of planning, a trained observer watched the surgeon carry out planning activities and used a stopwatch to record the time required from beginning to end, pausing the stopwatch any time an unavoidable interruption in planning occurred. This same procedure was followed for both traditional and online planning. The order of surgical planning (online software first vs traditional methods first) was randomized for each study patient, and the two planning sessions for each eye were separated by at least one hour to prevent memory effects influencing the time spent planning. To further reduce recall of the first planning session for each eye, the order of patients undergoing planning was also shuffled. For example, in a session where five eyes underwent surgical planning, each eye was randomized as to Veracity vs traditional method first, and the second planning session for the same eyes was undertaken at least an hour later with the order shuffled.

For the traditional method of planning, when either the Barrett True $\mathrm{K}$ or Barrett toric formula was needed for post-refractive eyes or those requiring toric correction, they were accessed via the website of the Asia Pacific Association of Cataract and Refractive Surgeons with the surgeon transferring the data from the biometry printout to the web interface and an observer recording the number of individual data points transferred to address the secondary outcome measure. For example, the keratometry value $44.25 @ 90^{\circ}, 44.75 @ 180^{\circ}$ would be considered four data points. With the online planning software, these formulas natively reside in the software and are populated by the measurements downloaded digitally from the biometer. The total time spent planning each eye by each method, as well as the number of data points manually transferred from one location to another, were recorded by a third-party observer during all planning sessions.

Previously published studies of error rates in the transcription of medical data in laboratory and other clinical settings have shown an error rate ranging from $2 \%$ to $5 \%$. In other words, out of every 100 data points manually transcribed in a medical setting, 2 to 5 of those data points have been shown to contain an error. For this study, we assumed a blended rate of $3.5 \%$ to do a calculation for the likelihood of a hypothetical eye having an error in data
Table I Patient Characteristics and Procedures Performed

\begin{tabular}{|l|l|}
\hline & Total \\
\hline N & 40 \\
Age & $69.3 \pm 13.3$ (range 53-85) \\
Females & $21(52 \%)$ \\
Right Eyes & $23(58 \%)$ \\
Post Refractive & $9(22.5 \%)$ \\
Required Astigmatic Planning & $20(50 \%)$ \\
\hline
\end{tabular}

transcription based on the number of data points transcribed for each method of planning. ${ }^{5-8}$

Following surgery, all patients underwent a manifest refraction 20-35 days after surgery. The spherical equivalent of this refraction was calculated and compared to the predicted spherical equivalent for the formula used preoperatively by each method for each eye. Since the same formulas were used in both traditional and online planning methods, no differences would be anticipated assuming no errors in data transcription were made.

\section{Results}

$\mathrm{IOL}=$ intraocular lens; $\mathrm{LRI}=$ limbal relaxing incision

A total of 40 patients were enrolled in this study over a 4-month period. The prolonged recruitment period was the result of the clinic and surgical operations of the enrolling surgeon being halted for two months due to the coronavirus pandemic (Table 1).

Of the 40 patients enrolled, 19 (48\%) were male and 21 $(52 \%)$ were female. The age of patients ranged from 53 to 85 years (mean $69.3 \pm 13.3$ ). The right eye was enrolled in $23(58 \%)$ of the enrolled patients. Of these patients, 9 $(22.5 \%)$ were post-refractive, and $20(50 \%)$ required astigmatic planning.

\section{Primary Outcome}

Average surgical planning time (seconds) was recorded for both the traditional EHR/paper method and for the online software method. With the traditional method, mean time to plan post-refractive cases was $533 \pm 192$ seconds vs $65 \pm 30$ seconds for the online software - a difference of 8 minutes per eye, or a time savings of $88 \%$. For non-post-refractive eyes, mean time for traditional planning was $153 \pm 60$ seconds vs $62 \pm 32$ seconds with online software a difference of 1.5 minutes, or a time savings of $60 \%$. For the combined groups, the mean planning time by the traditional method was $239 \pm 190$ seconds vs $63 \pm 31$ seconds with online software - a savings of 1.5 minutes or $60 \%$ 


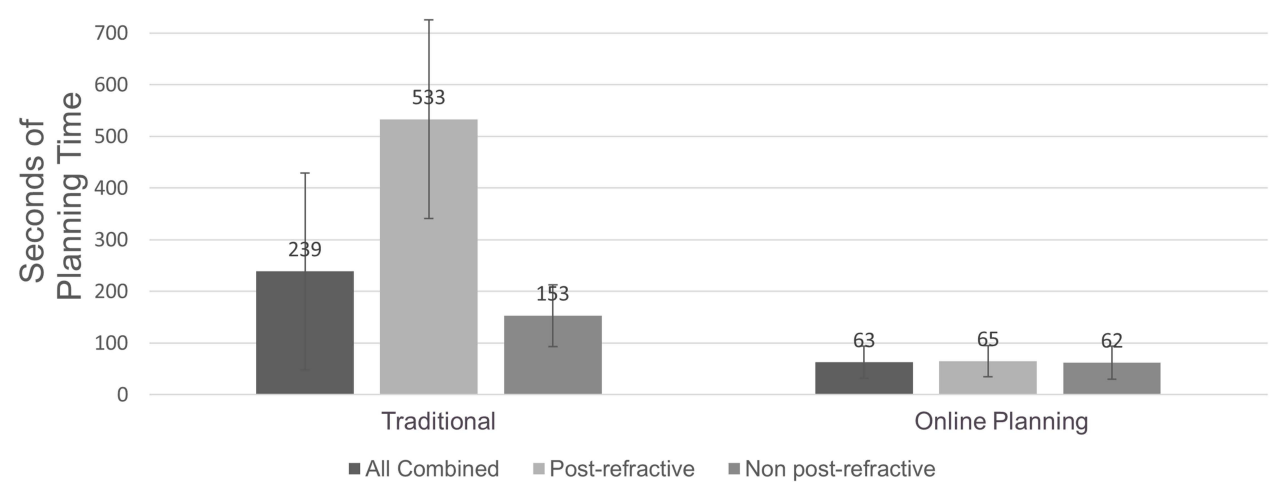

Figure I The mean surgical planning time was significantly reduced for post refractive $(P<0.0000 \mathrm{I})$, non post-refractive $(\mathrm{P}<0.0000 \mathrm{I})$, and combined patients $(\mathrm{P}<$ $0.00001)$.

(Figure 1). Each of these differences was statistically significant $(\mathrm{P}<0.00001$, paired $t$-test for all comparisons).

\section{Secondary Outcomes}

Manual transcription of data points was also assessed for both techniques. Among post-refractive patients, an average of $19.0 \pm 4.8$ (range 13-27) data points were manually transcribed using traditional documentation, versus a mean of $1.3 \pm 1.8$ (range $0-4$ ) data points with online planning (P $<0.00004$, paired $t$-test), a difference of 93\% (18 data points). Among non-post-refractive patients, an average of $3.5 \pm 1.6$ (range 1-6) data points were transferred via traditional documentation, versus a mean of $0.8 \pm 1.7$ (range 0 to 6) data points with online planning ( $\mathrm{P}<0.0000001$, paired $t$-test), or a difference of $77 \%$ ( 2.5 data points). For the entire study population, traditional documentation had a mean of $7.2 \pm 7.2$ (range 1-27) data points compared with a mean of $0.9 \pm 1.7$ (range 0 to 6 ) data points transferred using online planning, a percent difference of $(\mathrm{P}<0.0000001$, paired $t$-test), a difference of $88 \%$ (6.1 data points) (Figure 2).

Based on the assumption of a $3.5 \%$ rate of error in manual transcription of data points and the number of data points transcribed for each method, the per eye estimated likelihood of error in planning post-refractive patients using traditional methods was 2 in 3 eyes vs 1 in 22 eyes for the online software method. For non-post refractive patients, the likelihood of error using traditional methods was 1 in 8 eyes vs 1 in 36 eyes for the online software (Table 2, Figure 3).

Using actual refractive outcomes data in this sample of patients and comparing to the predicted spherical equivalent refraction from biometry by each method, no significant differences were noted for refractive accuracy between the IOL chosen using traditional documentation vs online software. Intended postoperative manifest spherical equivalent refraction measured one month after procedure was found to be within $0.5 \mathrm{D}$ of the predicted value

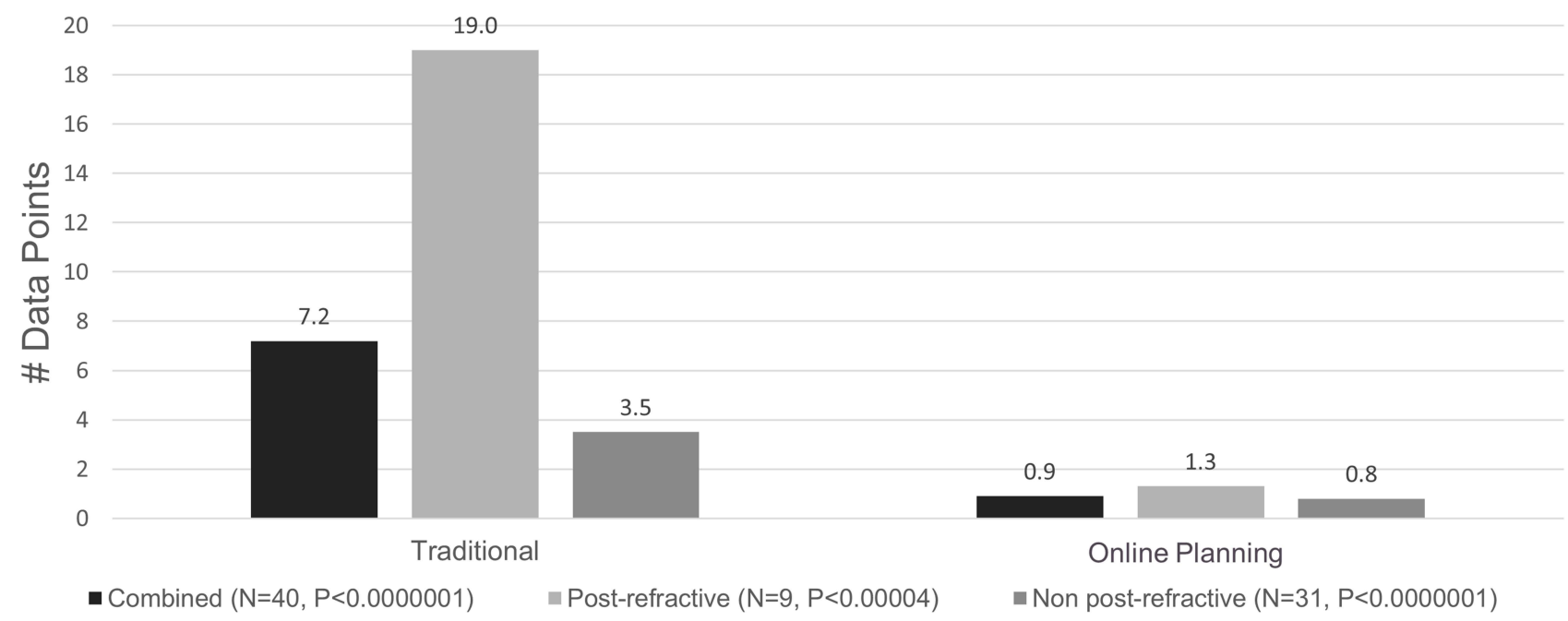

Figure 2 The mean manual transcription of data points was significantly reduced for post refractive $(P<0.00004)$, non post-refractive $(P<0.000000 \mathrm{I})$, and combined patients $(P<0.0000001)$. 
Table 2 Implications for Preventing Errors

\begin{tabular}{|l|l|l|l|l|}
\hline \multirow{2}{*}{} & \multicolumn{2}{|c|}{ Post-Refractive } & \multicolumn{2}{c|}{ Non Post-Refractive } \\
\cline { 2 - 5 } & \multicolumn{1}{|c|}{ Traditional } & \multicolumn{1}{|c|}{ Veracity } & \multicolumn{1}{c|}{ Traditional } & \multicolumn{2}{c|}{ Veracity } \\
\hline Error rate in transcribed data & $3.5 \%$ & $3.5 \%$ & $3.5 \%$ & $3.5 \%$ \\
Data points transcribed & 19 & 1.3 & 3.5 & 0.8 \\
Likelihood of error & 2 in 3 eyes & 1 in 22 eyes & 1 in 8 eyes & 1 in 36 eyes \\
\hline
\end{tabular}

for all enrolled patients, regardless of study arm. Thus, mean absolute prediction error was identical for both techniques (Figures 4 and 5).

\section{Discussion}

This study was conducted to investigate the efficiency and impact on accuracy of surgery with the use of a single cloud-based cataract surgery planning platform that electronically interfaces with diagnostic instruments and electronic health records (EHR) to obtain patient data. Because the system also contains modern lens planning formulas for astigmatism correction and post-refractive eyes in the same user interface, it aims to serve as a single platform for planning surgery. The online system was found to

\section{Likelihood of Erroneous Data Transcription}

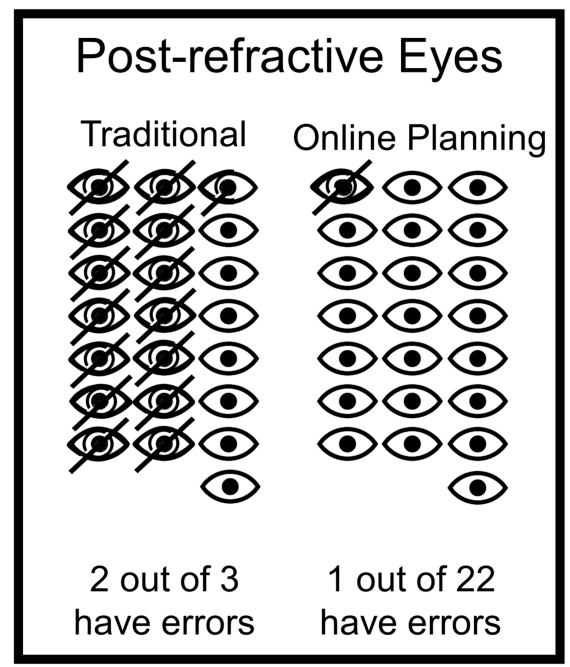

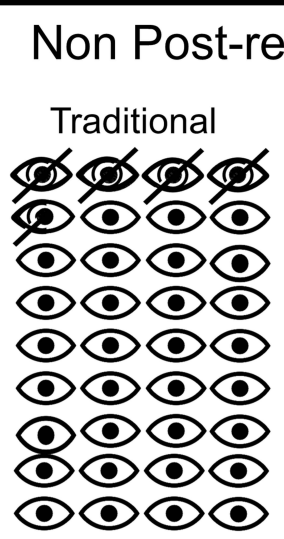

1 out of 8

have errors
Online Planning

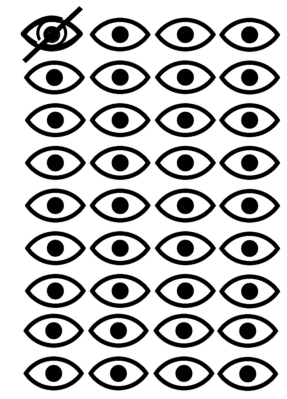

1 out of 36

have errors

Figure 3 Per eye estimated likelihood of error in post-refractive patients using traditional methods was 2 in 3 eyes vs $I$ in 22 eyes for the online software. Likelihood of error for non-post refractive patients using traditional methods was $I$ in 8 eyes vs $I$ in 36 eyes for the online software.

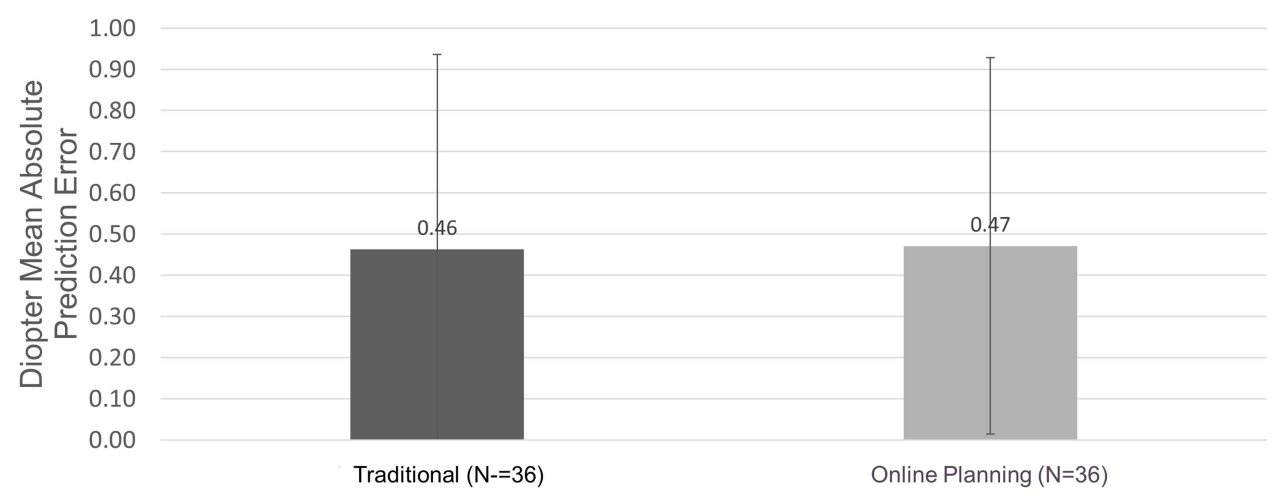

Figure 4 Refractive accuracy was roughly equivalent in both study arms, thus no significant differences were noted in mean absolute prediction error for traditional methods and online software. 


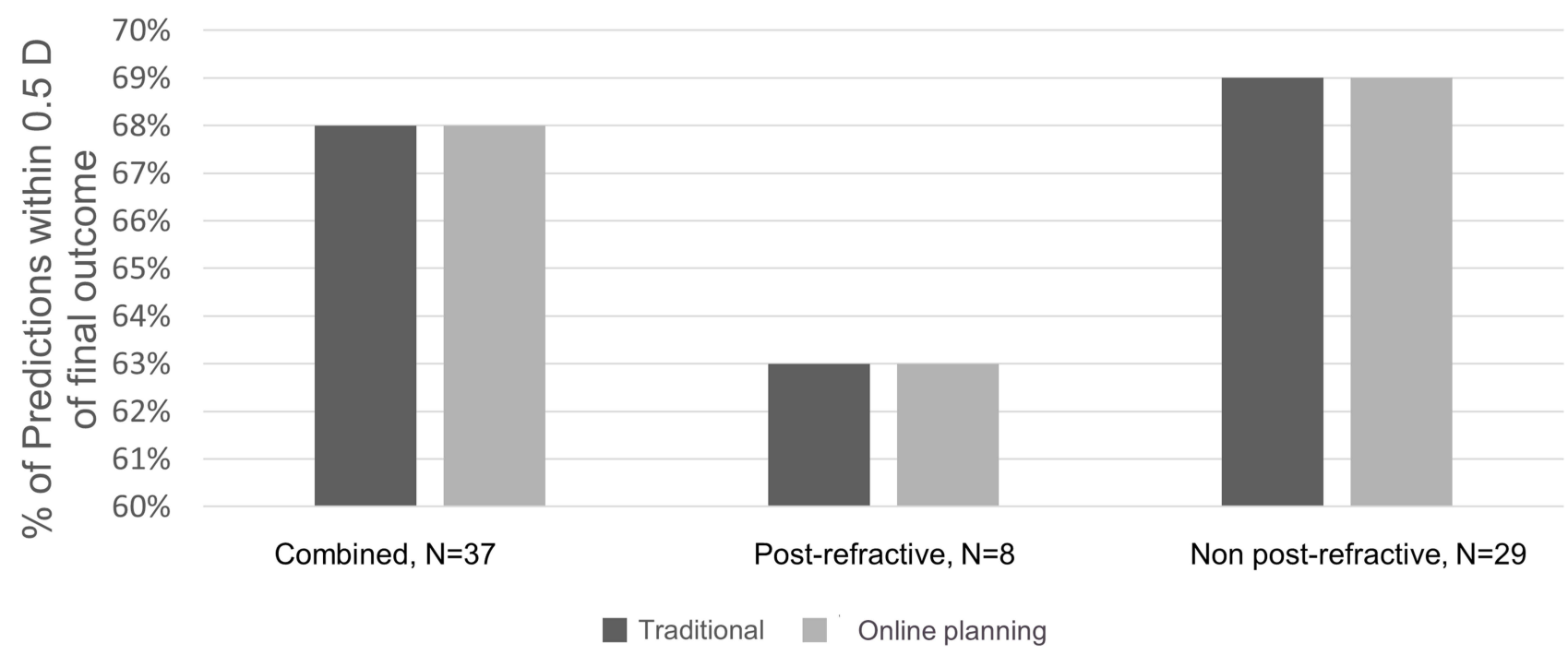

Figure 5 Intended postoperative manifest spherical equivalent refraction was found within $0.5 \mathrm{D}$ predicted value for all enrolled patients, thus no significant differences were noted between traditional methods and online software.

significantly reduce time required for surgical planning and the number of data points manually transcribed from one data source to another. No significant difference in absolute prediction error was observed between traditional planning and online in this study.

Reducing potential errors in cataract surgical planning is a worthwhile pursuit. Currently, cataract is highly prevalent in the United States, afflicting over 24.5 million Americans, with nearly 4 million cataract surgeries performed every year. ${ }^{9}$ The total number of persons with cataract is estimated to rise to 50 million by $2050 .^{9}$ Connected online software offers an avenue that is potentially more free of human error and more time efficient than current, manual methods of planning. In bypassing the need for online calculators and manual transcription of data, the use of the online planning system used in this study also reduced the time required for surgical planning by an average of $88 \%$ ( 8 minutes) per eye for postrefractive cases and $60 \%$ (1.5 minutes) per eye for nonpost refractive causes. A surgeon performing ten cases per week could potentially save thirty minutes per week or two hours per month if $10 \%$ of cases were post-refractive. This has important implications for improving the use of physician resources, given the projected growth of cataract surgery, as fewer surgeons can take care of more patients with theoretically higher quality care.

This study was not without limitations. First, this study was conducted by a single surgeon investigator. Since different surgeons may employ different surgical planning techniques, the time savings found in this study may vary from surgeon to surgeon. Also, in some practices, staff members may perform data transcription to save surgeons' time. Regardless of how much time is saved and for whom, it is important to acknowledge that the number of transcribed data points would be unlikely to vary significantly if this study were repeated in other practice settings. In decreasing the number of data points manually transcribed, and the associated likelihood of error by extension, integrated online planning software has the potential to benefit every surgeon and every practice.

In this study, $25 \%$ of the patients enrolled had undergone previous refractive surgery, and 50\% received IOLs or surgery requiring astigmatism planning. Both of these are higher rates than observed in most cataract practices. A more typical blend of patients might show less obvious benefits to online planning, but this higher mix of complex patients was chosen to evaluate whether the most challenging patients benefited more from online planning.

This study did not demonstrate a difference in the refractive accuracy of surgical planning by either method tested. One would expect that if a significant number of transcription errors occurred in either arm, a difference would be found in the recommended lens implant power. If such transcription errors occurred, they did not appear to affect the final refractive outcome because both tested methods showed identical refractive accuracy. Because previous medical literature suggests an error rate from $2 \%$ to $5 \%$ for transcribing data, we assumed a similar error rate might be observed in this study. Based on the observed number of transcribed data points, this would 
present the possibility of an error in 1 in 3 post-refractive eyes and 1 in 8 non-post-refractive eyes. Had this rate of transcription error occurred, it is likely a difference in refractive accuracy would have been observed between the two methods. This lower rate of observed errors may be due to double and triple checking of transcribed data points that is customary in the investigator's practice. While double and triple checks can mitigate inaccuracy of manual transcription, they are performed at a cost of time, and they do not guarantee freedom from errors. Even in a meticulous practice setting, a larger study would likely show the effect of errors, which would be more likely in manual planning, where more data points are transcribed. Even though this study did not demonstrate superior accuracy of online planning vs traditional methods, it stands to reason that reducing the number of manually transcribed data points represents an improvement in the quality of a process as important as IOL implant planning.

Recent technological advances in cataract surgery have provided patients with ever-improving visual outcomes with fewer complications, yet the methods used by surgeons for managing the information needed for surgical planning have changed very little over the same time period. Further study is warranted for systems that are designed to reduce the variability of surgery while reducing the effort of surgical practices to achieve the refractive desires of patients.

\section{Data Sharing Statement}

No deidentified participant data is being disclosed in this manuscript. No other study documents or participant information will be released.

\section{Acknowledgments}

Leslie Lemieux assisted in the collection of data in this study.

\section{Funding}

This study was funded by a grant from Alcon.

\section{Disclosure}

Miss Tarika Gujral has nothing to disclose. Dr John Hovanesian reports grants, personal fees from Zeiss, during the conduct of the study.

\section{References}

1. Congdon N, Vingerling JR, Klein BEK, et al; Eye Diseases Prevalence Research Group. Prevalence of cataract and pseudophakia/aphakia among adults in the United States. Arch Ophthalmol. 2004;122 (4):487-494.

2. Gimbel HV, Dardzhikova AA. Consequences of waiting for cataract surgery. Curr Opin Ophthalmol. 2011;22(1):28-30. doi:10.1097/ ICU.0b013e328341425d

3. Zeiss. IOL Master 700 Central Topography. Haag-Streit. LENSTAR; 2020.

4. Ferreira TB, Ribeiro F. How can we improve toric intraocular lens calculation methods? Current Insights. Clin Ophthalmol. 2020;14:1899-1908. doi:10.2147/OPTH.S238686

5. Mays JA, Mathias PC. Measuring the rate of manual transcription error in outpatient point-of-care testing. $J$ Am Med Inform Assoc. 2019;26(3):269-272. doi:10.1093/jamia/ocy170

6. McSwiney RR, Woodrow DA. Types of error within a clinical laboratory. J Med Lab Technol. 1969;26(4):340.

7. Tuckerman JF, Henderson AR. The clinical biochemistry laboratory computer system and result entry: validation of analytical results. Comput Methods Programs Biomed. 1985;20(1):103-116. doi:10.1016/0169-2607(85)90050-1

8. Wang L, Koch DD. Intraocular lens power calculations in eyes with previous corneal refractive surgery. J Ophthalmol. 2020. doi:10.1016/j. ophtha.2020.06.054

9. Erie JC. Rising cataract surgery rates: demand and supply. Ophthalmology. 2014;121:2-4.
Clinical Ophthalmology

\section{Publish your work in this journal}

Clinical Ophthalmology is an international, peer-reviewed journal covering all subspecialties within ophthalmology. Key topics include: Optometry; Visual science; Pharmacology and drug therapy in eye diseases; Basic Sciences; Primary and Secondary eye care; Patient Safety and Quality of Care Improvements. This journal is indexed on PubMed
Dovepress

Central and CAS, and is the official journal of The Society of Clinical Ophthalmology (SCO). The manuscript management system is completely online and includes a very quick and fair peer-review system, which is all easy to use. Visit http://www.dovepress.com/ testimonials.php to read real quotes from published authors. 